\title{
THE METABOLISM OF SPECIFICALLY LABELLED LACTATE AND PYRUVATE BY TWO-CELL MOUSE EMBRYOS
}

\author{
R. G. WALES AND D. G. WHITTINGHAM* \\ Department of Veterinary Physiology, University of Sydney, \\ Sydney 2006, N.S.W., Australia
}

(Received 13th March 1972)

Summary. The accumulation and utilization of substrate carbon by two-cell mouse embryos incubated in media containing combinations of lactate and pyruvate were studied. The results were consistent with utilization of the two substrates by common metabolic pathways. The intracellular accumulation of substrate carbon was greater when pyruvate as well as lactate was present in the medium. The evidence also suggests that pyruvate is more effective in maintaining the activity of the tricarboxylic acid cycle than lactate alone.

Fractionation of substrate carbon accumulated in embryos during a 30 -min incubation period indicated that approximately $10 \%$ of this carbon had entered the acid-insoluble (protein) fraction. This accumulation was increased substantially when a combination of lactate and pyruvate, rather than either substrate alone, was present in the incubation medium.

The major organic acids found to accumulate intracellularly from substrate were malate and citrate. The intracellular accumulation of lactate was confined to embryos incubated in medium containing this substrate. Base-containing compounds accounted for a substantial portion of the substrate carbon in embryos. Glutamic acid was the major amino acid identified in this component. Alanine accumulated from pyruvate or its combination with lactate but not from lactate alone. Small amounts of aspartate accumulated from both substrates.

The effect of the interaction of lactate and pyruvate on the development of two-cell mouse embryos to the blastocyst stage was confirmed. Although pyruvate was effective over a limited range of concentration, the effective range of concentration of lactate was not as limited. The present studies suggest that the beneficial effect of combinations of lactate and pyruvate could be due to a stimulation of the metabolism of the embryos and a more favourable level of macromolecule synthesis.

\section{INTRODUCTION}

The importance of the provision of certain exogenous energy substrates for the development of the preimplantation mouse embryo in vitro has been clearly

* Present address: Physiological Laboratory, Cambridge CB2 3EG, England. 
defined (Brinster, 1965a, b; Biggers, Whittingham \& Donahue, 1967). Studies using specifically labelled lactate and pyruvate have shown that there is no difference in the ability of one- and two-cell mouse embryos to accumulate and utilize these substrates when they are present individually as the sole energy source in the medium (Wales \& Whittingham, 1967). Nevertheless, the greater turnover of pyruvate, indicated by the production of $\mathrm{CO}_{2}$ and intracellular accumulation of the products of metabolism, probably enables pyruvate but not lactate to support the first cleavage division in vitro. Chemical analysis of the oviducal fluid of the rabbit has revealed the presence of both lactate $(2.5 \mathrm{~mm})$ and pyruvate $(0.2 \mathrm{~mm})$ (Holmdahl \& Mastroianni, 1965). Moreover, Brinster (1965b) found that these two substrates employed together in the medium were significantly better at maintaining the development of two-cell mouse embryos to the blastocyst stage in vitro than either alone.

Brinster (1965b) suggests that this effect of interaction between substrates on embryonic development may be caused by changes in the oxidation-reduction potential of the embryo. This in turn might favour particular metabolic pathways and so vary the carbon pools of the embryo into which substrate is channelled. In this way, a level of metabolic control could be maintained over development. Therefore, in this investigation, the short-term uptake and accumulation of substrate carbon from specifically labelled lactate and pyruvate were studied when the substrates were employed either alone or in combination in the incubation medium. A closer examination of the optimal combination of these substrates to support development of two-cell mouse embryos was also made, since the lactate concentration found in oviducal fluid (Holmdahl \& Mastroianni, 1965; Restall \& Wales, 1966) and blood serum (Long, 1961) is much lower than that used by Brinster (1965b) to culture mouse embryos in vitro.

\section{MATERIALS AND METHODS}

\section{General}

The procedures for the collection of two-cell mouse embryos from superovulated random-bred Swiss mice, 46 to $48 \mathrm{hr}$ after the injection of HCG, have been adequately described elsewhere (Brinster, 1963; Biggers, Whitten \& Whittingham, 1971). The medium used for the collection of embryos was a modified Krebs-Ringer bicarbonate solution containing 25 mM-DL-sodium lactate, $0.25 \mathrm{~mm}$-sodium pyruvate, $1 \mathrm{mg}$ bovine serum albumin $/ \mathrm{ml}, 60 \mu \mathrm{g}$ penicillin $/ \mathrm{ml}$ and $50 \mu \mathrm{g}$ streptomycin sulphate $/ \mathrm{ml}$ (Brinster, 1965b). The embryos were washed in two changes of similar medium devoid of energy substrates ( $2 \mathrm{ml} /$ wash) before incubation in a medium containing isotopically labelled or unlabelled substrates. When media contained combinations of lactate and pyruvate, the osmolarity was kept at approximately 0.308 osmol by adjusting the sodium chloride content of each medium.

\section{Isotopes}

Specifically labelled $\left[{ }^{14} \mathrm{C}\right]$ sodium pyruvate (Amersham Laboratories) was stored in small aliquots at $-40^{\circ} \mathrm{C}$ until use (Wales \& Whittingham, 1971). 
Initially, the dried aliquots were reconstituted with substrate-free medium to give a concentration of $1 \mathrm{~mm}$-pyruvate. This medium was further diluted with equal parts of either a substrate-free medium or one containing non-radioactive lactate to give a final concentration of $0.5 \mathrm{~mm}$-pyruvate. The specific activities of the pyruvate were 11.8 to $15.2 \mu \mathrm{Ci} / \mu \mathrm{mol}$ for $\left[1-{ }^{14} \mathrm{C}\right]$ sodium pyruvate and 11.9 to $17.5 \mu \mathrm{Ci} / \mu \mathrm{mol}$ for $\left[2-{ }^{14} \mathrm{C}\right]$ sodium pyruvate. Specifically labelled $\left[{ }^{14} \mathrm{C}\right]$ sodium DL-lactate (Amersham Laboratories) was diluted with medium to give the required concentration and specific activity. The final concentration of sodium DL-lactate in the medium and the specific radioactivity of the lactate were varied to meet the particular requirements of the experiment and the values are given in the relevant sections of the results.

In view of the high concentration and specific activity of isotope required in some experiments, a preliminary test was undertaken to determine whether exposure for $30 \mathrm{~min}$ to high levels of isotope had a detrimental effect on subsequent development. Two-cell mouse embryos were incubated for $30 \mathrm{~min}$ in media containing specifically labelled lactate at concentrations of 25 and $0.5 \mathrm{~mm}$ and at specific activities of 5.7 and $26.0 \mu \mathrm{Ci} / \mu \mathrm{mol}$, respectively, for $\left[1-{ }^{14} \mathrm{C}\right]$ lactate, and at 2.5 and $18.5 \mu \mathrm{Ci} / \mu \mathrm{mol}$, respectively, for $\left[2-{ }^{14} \mathrm{C}\right] \mathrm{lactate}$. At the completion of incubation, the embryos were washed twice by transfer through two changes of non-radioactive medium and then cultured for $72 \mathrm{hr}$ in medium containing unlabelled lactate $(25 \mathrm{~mm})$ and pyruvate $(0.5 \mathrm{~mm})$ as described by Brinster (1963). Of seventy-five embryos cultured/treatment, an average of $84 \%$ developed into blastocysts, compared with $81 \%$ for control samples of the same embryos, and development was not influenced by either the concentration or the specific activity of the isotope in the medium.

Authenticity and purity of the isotopically labelled substrates were checked by chromatography of samples of the media using liquid-liquid partition chromatography on a silicic acid support (O'Shea \& Wales, 1968).

\section{Accumulation of substrate carbon in embryos}

Depending on the experiment, between 50 and 250 embryos were placed in drops $(20 \mu \mathrm{l})$ of radioactive medium under $10 \mathrm{ml}$ paraffin oil contained in a plastic Petri dish. The embryos were incubated for $30 \mathrm{~min}$ at $37^{\circ} \mathrm{C}$ in the radioactive medium and then collected free from the incubation medium by centrifuging through isotonic sucrose (Wales \& Biggers, 1968). The isotonic sucrose contained unlabelled substrates in similar concentrations to the incubation medium, preliminary experiments having shown that this procedure helps to prevent loss of the radioactivity from the embryo during centrifugation. Each sample was assayed for radioactivity by liquid-scintillation techniques using $5 \mathrm{ml}$ of a Triton X-100: toluene mixture $(1: 2)$ containing $0.4 \% 2,5-$ diphenyloxazole (PPO) and $0.01 \%$ l,4-bis-(4-methyl-5-phenyloxazolyl-2) benzene (dimethyl POPOP) as scintillator. The accumulation of substrate carbon from the labelled site was calculated from the counts in the embryos and the specific activity of substrate in the incubation medium.

\section{Production of carbon dioxide}

The technique for measuring the production of $\mathrm{CO}_{2}$ by mouse embryos 
from labelled substrates has been described (Wales \& Biggers, 1968). Between 120 and 340 embryos/treatment were incubated in $\left[1-{ }^{14} \mathrm{C}\right]$ sodium pyruvate and $\left[1-{ }^{14} \mathrm{C}\right]$ sodium DL-lactate, and between 75 and 250 in $\left[2-{ }^{14} \mathrm{C}\right]$ sodium pyruvate and $\left[2{ }^{14} \mathrm{C}\right]$ sodium DL-lactate. Samples were acidified with $0 \cdot 1$ M-citrate buffer ( $\mathrm{pH} 4.0$ ) after incubation for $3 \mathrm{hr}$ at $37^{\circ} \mathrm{C}$ (Wales \& Whittingham, 1967) and the $\mathrm{CO}_{2}$ evolved was absorbed in $0.2 \mathrm{ml} 1 \mathrm{~N}$-sodium hydroxide. The sodium hydroxide was transferred to a scintillation vial $24 \mathrm{hr}$ later and the radioactivity was assayed by the liquid-scintillation method described above. Control samples of medium were incubated with the samples containing embryos and the amount of radioactive $\mathrm{CO}_{2}$ collected from the controls was used to correct ${ }^{14} \mathrm{CO}_{2}$ production from samples containing embryos.

\section{Extraction of embryos and fractionation of metabolites}

Following incubation in radioactive medium for $30 \mathrm{~min}$, embryos were separated from the incubation medium by centrifuging through isotonic sucrose as outlined above. They were then recovered from the broken tip of the centrifuge tube and extracted as previously described (Wales \& Whittingham, 1970), after the addition of $50 \mu \mathrm{l}$ sheep serum to act as carrier for the radioactive compounds. The metabolites in the acid-soluble fraction were fractionated into basic, acidic and neutral compounds by applying aliquots of this fraction to ion-exchange resins (Wales \& Whittingham, 1970) and assaying the eluate for radioactivity by liquid-scintillation techniques. The separation employed leads to the elution of amino acids with the basic compounds and of all other organic acids with the acidic compounds. Organic acids in the acid-soluble fraction were separated by paper chromatography using butanol:acetic acid:water $(4: 1: 5)$ as solvent and by liquid-liquid partition chromatography using silicic acid as support (O'Shea \& Wales, 1968). The amino acids in the basic compounds from the ion-exchange resins were separated by high-voltage paper electrophoresis using acetic acid:formic acid: water buffer at $\mathrm{pH} 1.85$ (Atfield \& Morris, 1961) and phthalate buffer at $\mathrm{pH} 5.9$ (Block, Durrum \& Zweig, 1958). The identity of the amino acids was checked further by chromatography on Whatman No. 1 paper using phenol: water $(4: 1)$ as solvent.

\section{Statistical analysis}

Most experiments were repeated three to six times and the results were subjected to statistical analysis. Where the accumulation of substrate carbon and the production of carbon dioxide from labelled substrates were measured, logarithmic transformation of the data was used to equalize variances. In the experiments assessing the effects of lactate and pyruvate on the development of mouse embryos, the response, measured as the percentage of two-cell mouse embryos developing into blastocysts, was transformed to angles before performing statistical analysis.

\section{RESULTS}

Effects of different combinations of lactate and pyruvate on development

In the first two experiments, the effect of different combinations of pyruvate 
and lactate on the development of two-cell mouse embryos was examined. The concentrations employed in the culture medium were $0,2.0 \mathrm{~mm}, 10.0$ and 50.0 mm-lactate in combination with $0,0.1 \mathrm{~mm}, 0.3 \mathrm{~mm}$ and 0.9 mm-pyruvate. Ten two-cell embryos were cultured in each substrate combination for $72 \mathrm{hr}$ and the experiment was repeated four times. The results, expressed as the percentage of blastocysts obtained in each combination (Text-fig. 1), showed that there was a significant effect of both pyruvate and lactate and an interaction between the two. In the absence of pyruvate, responses were low even in the presence of $50.0 \mathrm{~mm}$-lactate. The addition of 0.1 and $0.3 \mathrm{~mm}$-pyruvate

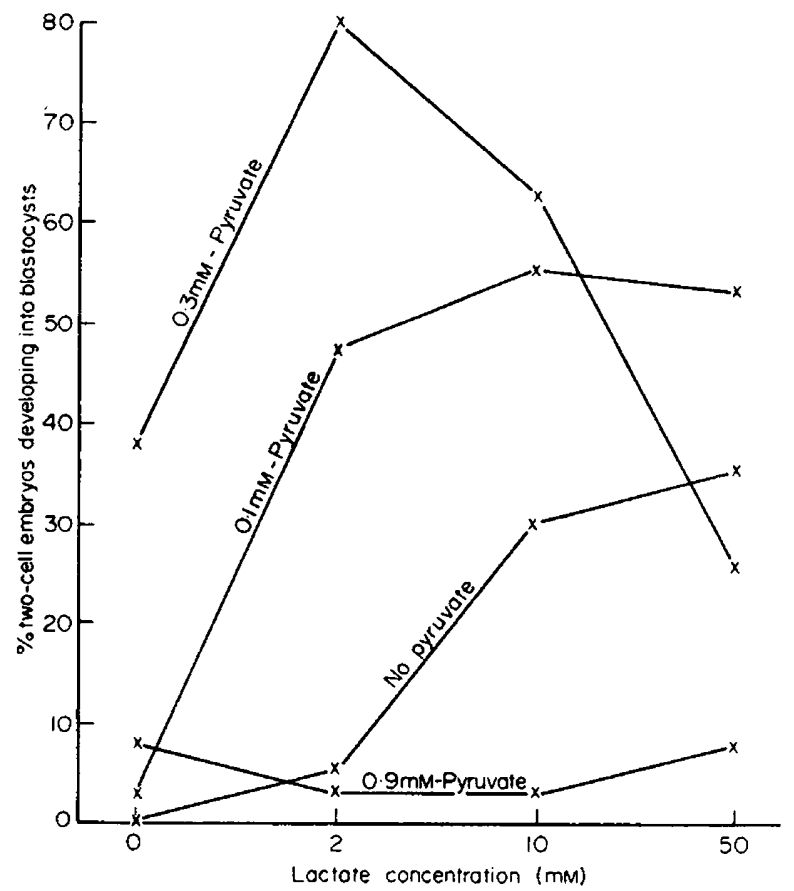

TEXT-FIG. 1. The development of two-cell mouse embryos in media containing varying concentrations of lactate and pyruvate.

to the medium improved responses, especially in combination with low concentrations of lactate, and the best response $(80 \%$ of embryos developing into blastocysts) was obtained when the medium contained $2.0 \mathrm{~mm}$-lactate and 0.3 mm-pyruvate. The highest concentration of pyruvate $(0.9 \mathrm{~mm})$ severely depressed development. A further experiment was carried out over a more restricted range of concentrations: $0.5 \mathrm{~mm}, 1.0 \mathrm{~mm}, 2.0 \mathrm{~mm}$ and $4.0 \mathrm{~mm}$-lactate and 0.1 $\mathrm{mm}, 0.2 \mathrm{~mm}, 0.3 \mathrm{~mm}$ and $0.4 \mathrm{~mm}$-pyruvate. The experiment was repeated six times and is graphically illustrated in Text-fig. 2. Again, there were significant effects of both lactate and pyruvate but over this restricted range of concentrations, no significant interaction between the two substrates was found. Overall, $4 \mathrm{~mm}$-lactate was more beneficial than the lower concentrations. Responses in media containing $0.1 \mathrm{~mm}$-pyruvate were low but near maximal responses were obtained with $0.3 \mathrm{~mm}$ and $0.4 \mathrm{~mm}$ pyruvate in combination with all concentrations of lactate. 
Accumulation of lactate and pyruvate alone and in combination

In a preliminary experiment to study the accumulation of substrate carbon by two-cell mouse embryos incubated in media containing lactate $(25 \mathrm{~mm})$ and pyruvate $(0.5 \mathrm{~mm})$ alone or in combination, it was found that the accumulation of carbon $1(\mathrm{C}-1)$ and carbon $2(\mathrm{C}-2)$ of lactate was significantly increased by the presence of pyruvate $(P<0.01)$, whereas the accumulation of $\mathrm{C}-1$ and C-2 of pyruvate was significantly decreased (fourfold) by the presence of 25.0

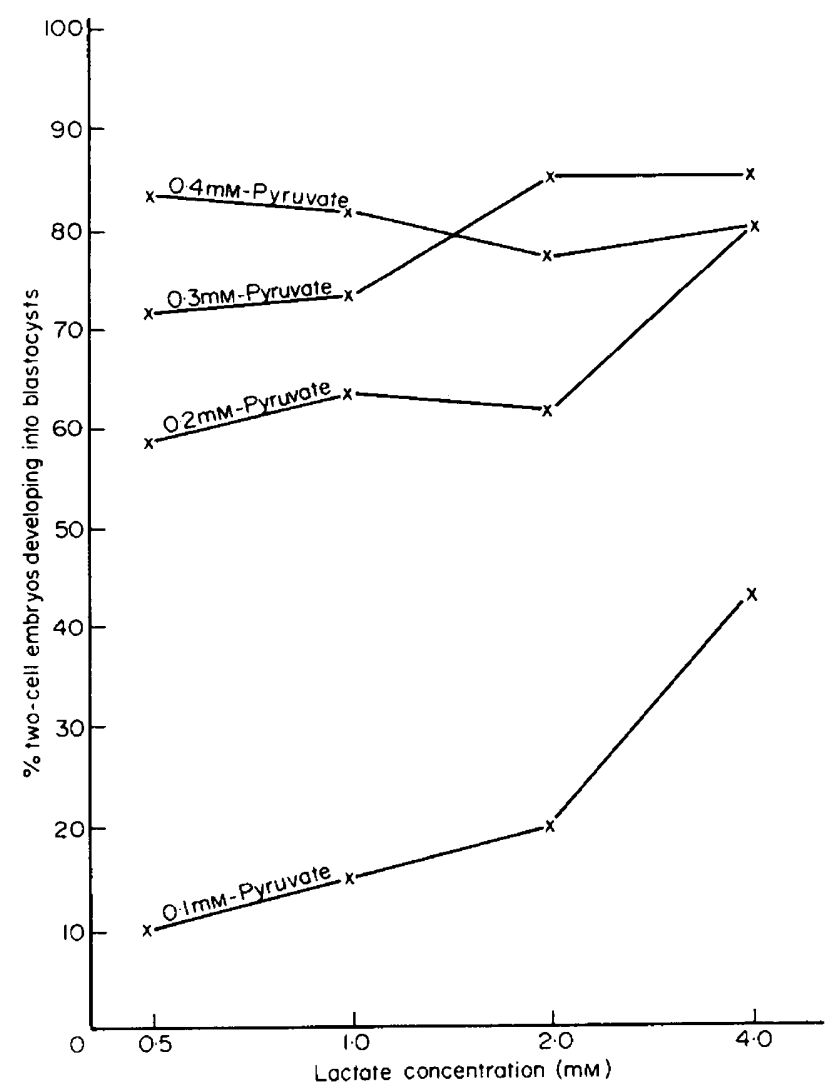

TexT-FIG. 2. The development of two-cell mouse embryos in the presence of a restricted range of concentrations of lactate and pyruvate.

mm-lactate $(P<0.001)$. In all cases, the accumulation of pyruvate carbon and the total substrate carbon accumulated from the combination of the substrates was greater than that from either substrate alone.

In view of these results and since a wide range of lactate concentrations supported development in the presence of pyruvate, further experiments were undertaken to investigate the effects of varying concentrations of lactate $(0.5$, 5.0 and $25.0 \mathrm{~mm}$ ), alone and in combination with $0.5 \mathrm{~mm}$-pyruvate, upon the accumulation of substrate carbon by two-cell mouse embryos during a 30 -min incubation period. To obtain an accurate assessment of substrate uptake from lactate in these experiments, the specific radioactivity of the lactate in the medium was varied with each treatment. For $\left[1-{ }^{14} \mathrm{C}\right]$ lactate, the specific 


\begin{tabular}{|c|c|c|c|c|}
\hline 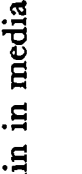 & 迹: & 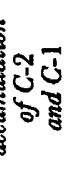 & 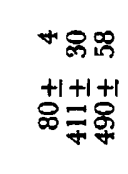 & 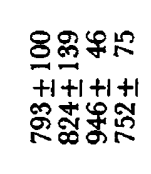 \\
\hline 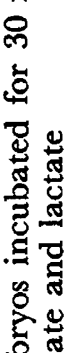 & 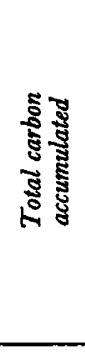 & తै & 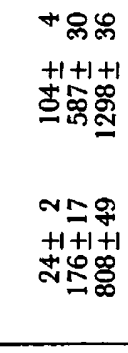 & 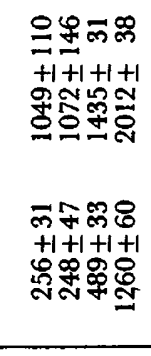 \\
\hline 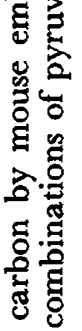 & 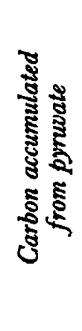 & త゙ & $|1|$ & 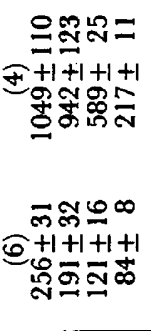 \\
\hline 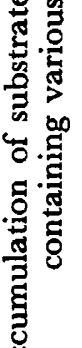 & 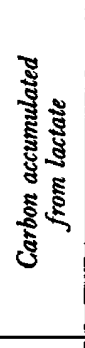 & ษૈ & 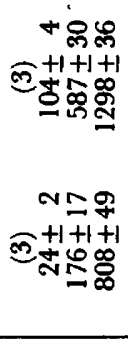 & 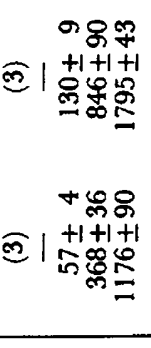 \\
\hline ع & 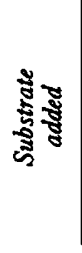 & 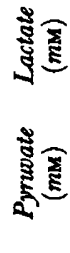 & ํํำ & 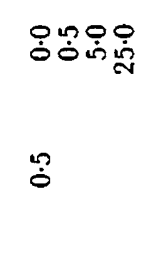 \\
\hline
\end{tabular}


activities at 25,5 and $0.5 \mathrm{~mm}$ were $5.7,8.7$ and $26.0 \mu \mathrm{Ci} / \mu \mathrm{mol}$, respectively. For $\left[2-{ }^{14} \mathrm{C}\right]$ lactate, the corresponding specific activities were $2 \cdot 5,5 \cdot 0$ and $18 \cdot 5$ $\mu \mathrm{Ci} / \mu \mathrm{mol}$. The results of the experiments are summarized in Table 1 .

Increase in the concentration of lactate in the absence of pyruvate increased the uptake of C-1 of lactate by approximately $40 \mathrm{fg}$ atoms/embryo for each $1 \mathrm{~mm}$ increase in the concentration of lactate. At all concentrations, accumulation of C-2 of lactate was greater than that of $\mathrm{C}-1$ but its value was not as closely related to the concentration of lactate in the medium as was that for C-1. The addition of pyruvate caused a substantial rise in the uptake of lactate at all concentrations of lactate studied.

As in the earlier experiments, the accumulation of $\mathrm{C}-2$ of pyruvate in the absence of lactate was approximately four times as great as that of C-1 of pyruvate. The addition of equimolar amounts of lactate to the medium caused a slight fall in the accumulation of C-1 and C-2 of pyruvate. As the concentration of lactate increased, there was a progressive decrease in the amount of substrate carbon accumulated from both C-1 and C-2 of pyruvate.

By combining the data for lactate and pyruvate uptake, estimates of the total uptake of substrate carbon from media containing varying concentrations of lactate in combination with pyruvate can be made and these are included in Table 1. With 25 and 5 mm-lactate in combination with $0.5 \mathrm{~mm}$-pyruvate, total substrate carbon accumulation from C-1 or C-2 was greater than the accumulation of substrate when either substrate alone was added to the medium. With 0.5 mm-lactate, combined uptake from lactate and pyruvate was similar to that from pyruvate alone. Major variations in the total uptake under these circumstances appear to be due mainly to an increase in the accumulation of substrate which has not been decarboxylated, as the amount of decarboxylated substrate in the embryos, estimated by the difference in the accumulation of C-2 and C-1 of the substrates, remained relatively constant in all combinations and was similar to that with pyruvate alone. In the absence of pyruvate, the accumulation of decarboxylated substrate was low and was influenced by the concentration of lactate in the medium.

\section{Oxidation of lactate and pyruvate by mouse embryos}

The production of carbon dioxide from specifically labelled pyruvate in the presence of different concentrations of non-radioactive lactate was measured and the results are presented in Table 2. Production of carbon dioxide from C-1 of pyruvate was approximately three times that from C-2 of pyruvate, and the ratio $\left(\mathrm{CO}_{2}\right.$ from $\mathrm{C}-1$ of pyruvate $) /\left(\mathrm{CO}_{2}\right.$ from $\mathrm{C}-2$ of pyruvate) was not significantly affected by the presence or absence of lactate. Equimolar amounts of lactate added to the medium caused a slight $(20$ to $25 \%)$ decrease in the pyruvate and substantially higher concentrations of lactate were required to cause a marked depression in the utilization of pyruvate.

The production of carbon dioxide from specifically labelled sodium lactate at concentrations of $0.5 \mathrm{~mm}, 5 \mathrm{~mm}$ and $25 \mathrm{~mm}$ in combination with $0.5 \mathrm{~mm}$ pyruvate was also measured and the results are included in Table 2. When equal amounts of lactate and pyruvate were present, only a small amount of C-1 of lactate was converted to carbon dioxide. As the concentration of lactate 
rose, its oxidation increased and compensated for the fall in oxidation of pyruvate observed in the previous experiment. As with pyruvate, the production of carbon dioxide from $\mathrm{C}-1$ of lactate was two to three times that from $\mathrm{C}-2$ of lactate.

Entry of substrate carbon to the intracellular carbon pools of the embryo

Experiments were undertaken to determine the carbon pools into which pyruvate and lactate, either alone or in combination, entered during a 30 min period of incubation. Two-cell embryos were incubated in media containing $5 \mathrm{~mm}$-lactate, $0.5 \mathrm{~mm}$-pyruvate or a combination of $5 \mathrm{~mm}$-lactate

Table 2. The production of carbon dioxide from specifically labelled substrate by mouse embryos incubated for $3 \mathrm{hr}$ in media containing various concentrations of lactate in combination with pyruvate

\begin{tabular}{|c|c|c|c|c|c|}
\hline \multicolumn{2}{|c|}{ Substrate added } & \multirow{2}{*}{$\begin{array}{l}\text { Position } \\
\text { labelled }\end{array}$} & \multicolumn{3}{|c|}{$\begin{array}{c}\mathrm{CO}_{2} \text { production from position labelled } \\
(\text { fmol/embryo/3 hr })\end{array}$} \\
\hline $\begin{array}{l}\text { Pyruvate } \\
(\mathrm{mM})\end{array}$ & $\begin{array}{l}\text { Lactale } \\
(m \mathrm{M})\end{array}$ & & $\mathrm{CO}_{2} \underset{\text { pyruvate }}{\text { from labelled }}$ & $\mathrm{CO}_{2}$ from labelled & Total $\mathrm{CO}_{2}$ \\
\hline 0.5 & $\begin{array}{r}0.0 \\
0.5 \\
5.0 \\
25.0\end{array}$ & C-2 & $\begin{array}{c}(3) \\
9300 \pm 1200 \\
7300 \pm 1700 \\
2800 \pm 100 \\
900 \pm 100 \\
(3) \\
3200 \pm 200 \\
2400 \pm 200 \\
1300 \pm 200 \\
400 \pm \quad 40\end{array}$ & $\begin{array}{c}(2) \\
800 \pm 100 \\
3400 \pm 300 \\
6300 \pm 1300 \\
- \\
1500 \pm 500\end{array}$ & $\begin{array}{l}9300 \pm 1200 \\
8100 \pm 1200 \\
6200 \pm 200 \\
7100 \pm 800 \\
3200 \pm 200 \\
2800 \pm 300\end{array}$ \\
\hline
\end{tabular}

Values are the means \pm S.E.M. and the number of replicates is given in parentheses at the head of each column of results.

plus 0.5 mm-pyruvate and the entry of both C-1 and C-2 of each substrate into the carbon pools of the embryo was estimated. To ensure sufficient incorporation of isotope for the fractionation, specific activities of $13 \cdot 0 \mu \mathrm{Ci} / \mu \mathrm{mol}$ for $\left[1-{ }^{14} \mathrm{C}\right]$ lactate and $18.5 \mu \mathrm{Ci} / \mu \mathrm{mol}$ for $\left[2-{ }^{14} \mathrm{C}\right]$ lactate were used. Between 200 and 500 embryos were incubated in media containing $1-^{14} \mathrm{C}$-labelled substrate and between 125 and 275 embryos in $2{ }^{14} \mathrm{C}$-labelled substrate. This number ensured a total incorporation of between 1700 and $7000 \mathrm{~d} / \mathrm{min}$ of radioactivity for each batch of embryos. After $30 \mathrm{~min}$, the embryos were recovered and fractionated as outlined in the Materials and Methods section. The results for three replicates of the experiment are given in Table 3.

Between 90 and $95 \%$ of the substrate carbon incorporated into the embryos during this short period of incubation was found in the acid-soluble fraction. The remainder had entered the acid-insoluble fraction and there was no labelling of lipids. Accumulation of substrate carbon in both the acid-insoluble and acid-soluble fraction was greater when a combination of lactate and pyruvate was present in the incubation medium than when either substrate was added alone. This effect was due to an increased accumulation of both $\mathrm{C}-1$ and $\mathrm{C}-2$ of substrate. In addition, with lactate and pyruvate in combination, 


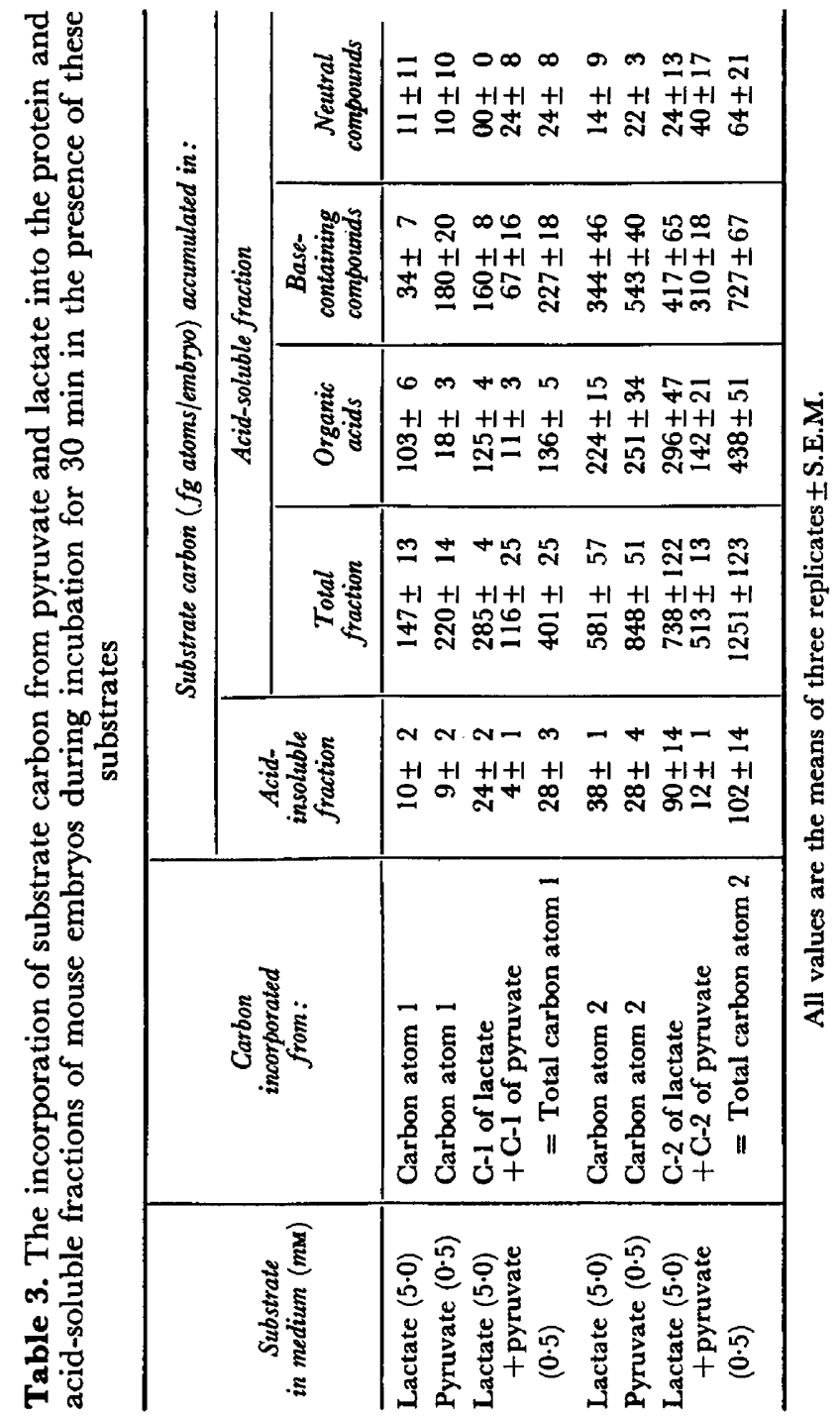


lactate contributed 60 to $90 \%$ of the total carbon incorporated. Fractionation of the acid-soluble material on ion-exchange resins showed major incorporation into both the base-containing and the acidic compounds. Incorporation into neutral compounds was small and inconsistent, isotope being found in this fraction in only six of the twelve samples assayed. Little accumulation of pyruvate occurred in the acidic compounds either with or without the addition of lactate. With lactate as sole energy source, this fraction accounted for most of the C-1 accumulated. Both lactate and pyruvate showed similar substantial incorporation of $\mathrm{C}-2$ into the acidic compounds. However, the accumulation of decarboxylated substrate measured as the difference between C-2 and C-1, was greater after incubation in pyruvate $(230 \mathrm{fg}$ atoms/embryo) than after incubation in lactate (120 fg atoms/embryo) and was greatest when a combination of the substrates was used ( $300 \mathrm{fg}$ atoms/embryo). With pyruvate alone, the major portion of the C-1 which accumulated in the embryo was found in the base-containing compounds. On the other hand, almost no C-1 of lactate accumulated in basic compounds unless pyruvate was also added to the medium. Substantial quantities of C-2 accumulated in these compounds from both lactate and pyruvate. As with the acidic fraction, an estimate of the amount of decarboxylated product was made from the difference between the accumulation of $\mathrm{C}-2$ and $\mathrm{C}-1$. In this case, the accumulation of decarboxylated substrate was similar for incubations in pyruvate ( $360 \mathrm{fg}$ atoms/embryo) and lactate (310 fg atoms/embryo) but was substantially increased to $500 \mathrm{fg}$ atoms/ embryo following incubation in the combination of substrates.

The residue of the acid-soluble fraction was applied to silicic acid columns and the organic acids eluted. Lactate was the major carboxylic acid identified in incubations containing this substrate and accounted for approximately 100 fg atoms/embryo of both C-1 and C-2 of substrate accumulated in the acidic fraction under these conditions. In incubations using $2-{ }^{14} \mathrm{C}$-labelled substrates, a large proportion of label not accounted for by lactate was eluted from the columns at a position corresponding to malate or citrate. This accumulation of C-2 was greatest with pyruvate or its combination but was low with lactate alone. No further identification was possible in this experiment.

\section{Identification of specific metabolites accumulated from lactate and pyruvate}

Embryos ( 300 to $500 /$ treatment) were incubated for $30 \mathrm{~min}$ in media containing $2 \cdot{ }^{14} \mathrm{C}$-labelled lactate $(5 \mathrm{~mm})$ and pyruvate $(0.5 \mathrm{~mm})$ alone or in combination. The specific radioactivity of each substrate was adjusted to 17.5 $\mu \mathrm{Ci} / \mu \mathrm{mol}$. The acid-soluble component of the isotope accumulated in these embryos was separated on ion-exchange resins. The acidic compounds were reduced in volume and chromatographed on silicic acid as in the previous experiment or on Whatman No. 1 paper using butanol:acetic acid:water $(4: 1: 5)$ as solvent. The proportions of labelled lactate, malate and citrate in the fraction was then calculated. The base-containing compounds were subjected to high-voltage electrophoresis as described in the Materials and Methods section. Three labelled compounds were found in this fraction corresponding to glutamic acid, alanine and aspartic acid and accounted for over $90 \%$ of the labelled basic compounds. Radioactive peaks corresponding 


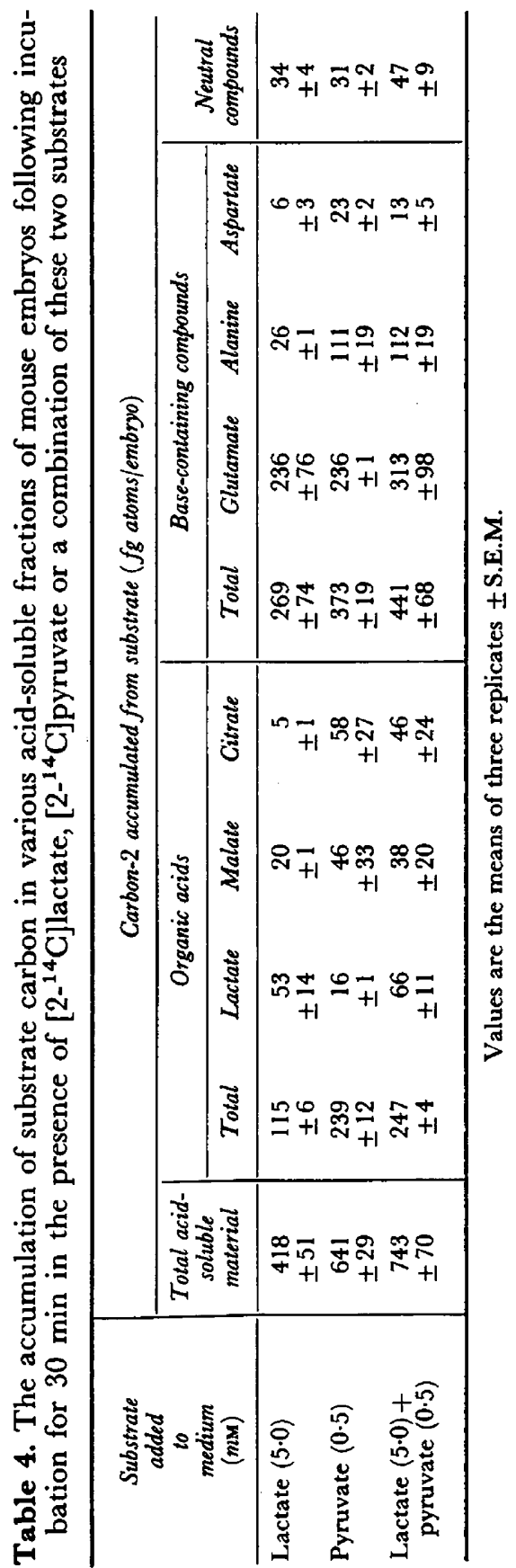


to these three amino acids were also obtained when further aliquots of this fraction were chromatographed in a descending system on Whatman No. 1 paper using phenol: water $(4: 1)$ as solvent.

The results for three replicates of the experiment are given in Table 4. In general, the pattern of uptake of substrate carbon from the three media into the acid-soluble fraction of embryos was similar to that found in the previous experiment. The smallest uptake was recorded when lactate alone was present in the medium and the largest occurred from the combination of lactate and pyruvate. As in the last experiment, the intracellular accumulation of substantial amounts of lactate was confined to those embryos incubated in media containing this substrate. Paper chromatography of the acid-soluble fraction allowed the separation of malate and citrate. With lactate alone, embryos accumulated small amounts of malate but not citrate. With pyruvate or its combination, both malate and citrate were found. On the average, more labelled citrate than malate accumulated under these circumstances. The amounts of substrate carbon found in both these intermediates varied considerably between replicates, however, and there was no significant difference in their accumulation. The three organic acids, lactic, malic and citric acids, accounted for $60 \%$ of the labelled acidic compounds in the embryos.

Glutamic acid was the major amino acid labelled in all embryos. There was a $30 \%$ rise in its average uptake when lactate and pyruvate were combined. The effect was not significant, however, because of large variations between replicates. Alanine accounted for 25 to $30 \%$ of the basic compounds accumulated from pyruvate or its combination. With lactate alone, little alanine was identified. A small amount of aspartic acid accumulated in all treatments.

\section{DISCUSSION}

The present results confirm the earlier observation of Brinster (1965b) that there is an interaction between these energy sources such that certain combinations of the two substrates give a better response than either substrate alone. Pyruvate is effective over a limited range of concentrations, similar to the levels previously found to be optimal for development (Brinster, 1965a, b; Whitten, 1971). The effective range of concentration of lactate is not as limited and a much lower range of concentrations $(0.5$ to $4.0 \mathrm{~mm})$ than previously examined by Brinster (1965b) can produce near optimal responses when in combination with 0.2 to $0.4 \mathrm{~mm}$ pyruvate. These latter levels of lactate and pyruvate approach those that have been measured in the oviducal fluid of the rabbit (Holmdahl \& Mastroianni, 1965) and more recently in the oviducal fluid of the mouse (W. J. Brunton \& R. L. Brinster, personal communication). They are, therefore, presumed to be close to the true physiological levels present during the development of mouse embryos in vivo.

When lactate and pyruvate are employed individually in the culture medium, the accumulation of radioactivity from substrate labelled at the C-1 and C-2 positions is of the same order as that reported previously (Wales \& Whittingham, 1967). The evidence from these earlier studies suggested that both lactate and pyruvate enter the embryo by diffusion, leading to the intracellular 
accumulation of the parent substrate. However, in the case of pyruvate, fractionation of the intracellular labelled compounds in the present experiment has shown that at least $80 \%$ of this label is found in the base-containing compounds rather than in the organic acids. Alanine was identified in sufficient quantity in the embryo to account for the major portion of this incorporation. Probably a small proportion of the incorporation also results from the fixation of carbon dioxide over this period (Quinn \& Wales, 1971). Thus, the accumulation of $\mathrm{C}-1$ of pyruvate during incubation at $37^{\circ} \mathrm{C}$ appears to be due mainly to the activity of the enzyme, alanine aminotransferase (EC 2.6.1.2) rather than substrate diffusion. The results, using varying concentrations of lactate both in the present experiment and in the earlier study (Wales \& Whittingham, 1967), show a linear relationship between lactate concentration and accumulation of C-1 of this substrate. Fractionation of the intracellular label indicated that most of this accumulation occurred as the parent substrate, but the intracellular concentration of substrate carbon accumulated from C-1 of lactate was always much lower than that present in the incubation medium. Whether the low intracellular concentration of lactate found in the present experiments is due to limited transfer of the substrate across the cell membrane (see Brinster, 1965a) or to loss from the embryo during recovery from the incubation medium is unknown and, at present, is under examination.

The isotopic studies using combinations of the two substrates indicate that there is a preferential utilization of pyruvate to lactate. Thus, there was less than a fivefold decrease in the intracellular accumulation of substrate carbon from pyruvate when the lactate concentration in the medium was fifty times greater than that of pyruvate. Results of this nature might be expected if the utilization of lactate occurs mainly by the oxidative conversion of lactate to pyruvate catalysed by lactate dehydrogenase (Brinster, 1965c). Under these circumstances, the reduction in the utilization of pyruvate would be related to the rate of conversion of lactate to pyruvate and the subsequent competition of the two sources of carbon for entry into the carbon pools of the embryo. In addition, the finding that the ratio $\left(\mathrm{CO}_{2}\right.$ from $\left.\mathrm{C}-1\right) /\left(\mathrm{CO}_{2}\right.$ from $\left.\mathrm{C}-2\right)$ for pyruvate and for lactate ranges from 2 to 3 and is unaffected by the presence or absence of the other substrate is further evidence that both substrates are utilized by common metabolic pathways.

The production of carbon dioxide from specifically labelled pyruvate is of the same order as that reported earlier (Wales \& Whittingham, 1967). The decrease in the production of carbon dioxide from pyruvate in the presence of varying concentrations of lactate parallels the effects on the accumulation of substrate carbon from pyruvate. However, when lactate is added, total oxidation of substrate carbon remains relatively constant. In this study, the production of carbon dioxide from substrate in a medium containing 25 mm-lactate and 0.5 mM-pyruvate was approximately twice that reported when lactate was present in the medium alone (Wales \& Whittingham, 1967). Thus, pyruvate or its combination with lactate is more effective in maintaining the activity of the tricarboxylic acid cycle than lactate alone. No doubt the finding that the intracellular accumulation of decarboxylated products such as malate and citrate is greater in the presence of pyruvate than in its absence is also linked with 
activity of the tricarboxylic acid cycle. Possibly the presence of pyruvate in the medium exerts its influence by changing the redox state of the cytoplasmic NAD-couple in the direction of oxidation. This, in turn, could influence the mitochondrial redox potential. The availability of both oxaloacetate and acetylCoA could thus be increased, leading to an acceleration of the citrate oxaloacetate lyase (EG 4.1.3.7.) reaction; a reaction which plays an important rôle in the regulation of the rate of the cycle (Krebs, 1970). In addition, the malate and citrate which were found to accumulate in these experiments may play a part in the regulation of the energy metabolism of the embryo. Their precise rôle is yet to be determined.

Fractionation of the isotope accumulated from specifically labelled substrates showed that a small incorporation of substrate carbon into the acid-insoluble fraction of embryos had occurred even after this short period of incubation. Substrate accumulation into this fraction probably represents incorporation of carbon into protein rather than macromolecules such as RNA at this early stage of development (Mintz, 1964; Monesi \& Salfi, 1967; Tasca \& Hillman, $1970)$. Although the incorporation into this fraction represents a small proportion of the total incorporation, a significant interaction was found between the two substrates. Thus, the combination of lactate with pyruvate led to a threefold increase in the accumulation of carbon in this fraction over that which occurred in the presence of either substrate alone. This effect is probably mediated by the amination of intermediates of the tricarboxylic acid cycle and the subsequent incorporation of the amino acids formed into the embryonic proteins. The accumulation of base-containing intermediates following decarboxylation of substrate showed a similar interaction between substrates to that found in the macromolecular fraction.

The small amount of substrate carbon incorporated after a 30 -min incubation and the limited number of embryos available for any one experiment considerably increases the problem of making an accurate quantitative assessment of the intracellular accumulation of labelled metabolites. This is reflected in the large standard errors of some of the estimates in Table 4. Notwithstanding these problems, the results do shed light on the metabolic pathways involved in substrate utilization. The accumulation of substrate carbon in glutamic acid accounts for 90 to $95 \%$ of the amino acids formed after substrate has been decarboxylated. The pattern of incorporation of substrate carbon into this compound agrees with that expected from the uptake of C-1 and C-2 of substrate into the basic compounds (see Table 3). Thus, it would appear that the conversion of oxoglutarate to glutamate is an important first step in the entry of substrate carbon to the synthetic apparatus of the developing embryo.

\section{AGKNOWLEDGMENTS}

The authors wish to express their thanks to Professor C. W. Emmens for the provision of facilities and helpful criticism of the manuscript. The work was aided by a grant from the Australian Research Grants Committee. One of us (D.G.W.) was supported by a University of Sydney Post Doctoral Fellowship. 


\section{REFERENCES}

Atrield, G. N. \& Morris, G. J. O. R. (1961) Analytical separations by high-voltage paper electrophoresis. Amino acids in protein hydrolysates. Biochem. 7. 81, 606.

Biggers, J. D., Whitten, W. K. \& Whittingham, D. G. (1971) The culture of mouse embryos in vitro. In: Methods of Mammalian Embryology, p. 86. Ed. J. C. Daniel. W. H. Freeman and Co, San Francisco and London.

Biggers, J. D., Whittingham, D. G. \& Donahue, R. P. (1967) The pattern of energy metabolism in the mouse oocyte and zygote. Proc. natn. Acad. Sci. U.S.A. 58, 560.

Block, R. J., Durrum, E. L. \& Zweig, G. (1958) A manual of paper chromatography and paper electrophoresis. Academic Press, New York.

Brinster, R. L. (1963) A method for in vitro cultivation of mouse ova from two-cell to blastocyst. Expl Cell Res. 32, 205.

Brinster, R. L. (1965a) Studies on the development of mouse embryos in vitro. II. The effect of energy source. F. exp. Zool. 158, 59.

BRINSTER, R. L. (1965b) Studies on the development of mouse embryos in vitro. IV. Interaction of energy sources. F. Reprod. Fert. 10, 227.

BRINSTER, R. L. (1965c) Lactic dehydrogenase activity in the preimplantated mouse embryo. Biochim. biophys. Acta, 110, 439 .

Holmdaht, T. H. \& Mastroianni, L. (1965) Continuous collection of rabbit oviduct secretions at low temperature. Fert. Steril. 16, 587.

KREBS, H. A. (1970) Rate control of the tricarboxylic acid cycle. In: Advances in Enzyme Regulation, Vol. 8, p. 335. Ed. G. Weber. Pergamon Press, New York.

Long, C. (Editor) (1961) Biochemist's handbook. D. Van Nostrand Co., Princeton, N.J.

Mintz, B. (1964) Synthetic processes and early development in the mammalian egg. J. exp. Zool. 157, 85.

Monesi, V. \& Salfi, V. (1967) Macromolecular synthesis during early development in the mouse embryo. Expl Cell Res. 46, 632.

O'Shea, T. \& WALes, R. G. (1968) Metabolism of $\left[1-{ }^{14} \mathrm{C}\right]$ sodium lactate and $\left[2-{ }^{14} \mathrm{C}\right]$ sodium lactate by ram spermatozoa. 7. Reprod. Fert. 15, 337.

Quinn, P. \& Wales, R. G. (1971) Fixation of carbon dioxide by pre-implantation mouse embryos in vitro and the activities of enzymes involved in this process. Aust. 7. biol. Sci. 24, 1277.

Restall, B. J. \& Wales, R. G. (1966) The fallopian tube of the sheep. III. The chemical composition of the fluid from the fallopian tube. Aust. F. biol. Sci. 19, 687.

TAsca, R. J. \& Hillman, N. (1970) Effects of actinomycin D and cyclohexamide on RNA and protein synthesis in cleavage stage mouse embryos. Nature, Lond. 225, 1022.

Wales, R. G. \& BigGers, J. D. (1968) The permeability of two- and eight-cell mouse embryos to Lmalic acid. F. Reprod. Fert. 15, 103.

Wales, R. G. \& Whitringham, D. G. (1967) A comparison of the uptake and utilization of lactate and pyruvate by one- and two-cell mouse embryos. Biochim. biophys. Acta, 148, 703.

Wales, R. G. \& Whitringham, D. G. (1970) Metabolism of specifically labelled pyruvate by mouse embryos during culture from the two-cell stage to the blastocyst. Aust. F. biol. Sci. 23, 877 .

Wales, R. G. \& Whitringham, D. G. (1971) Decomposition of sodium pyruvate in culture media stored at $5^{\circ} \mathrm{C}$ and its effects on the development of the preimplantation mouse embryo. $\mathcal{J}$. Reprod. Fert. 24, 126.

WhIrTEN, W. K. (1971) Nutrient requirements for the culture of preimplantation embryos in vitro. Schering Symposium on Intrinsic and Extrinsic Factors in Early Mammalian Development. Adv. Biosci. 6, 129. 